\title{
Radiographic Manifestations of Tuberculosis in HIV Positive Patients: Correlation with CD4 Positive Tcell Count
}

\author{
Mehrdad Bakhshayesh Karam,," Soheyla Zahirifard, and Farahnaz Aghahosseini ${ }^{2}$ \\ ${ }^{1}$ Masih Daneshvari Hospital, Pediatric Respiratory Diseases Research Center, Nat \\ ${ }^{2}$ Masih Daneshvari Hospital \\ "Corresponding author: Mehrdad Bakhshayesh Karam, Masih Daneshvari Hospital, Pediatric Respiratory Diseases Research Center, Nat. E-mail: \\ mehrdadbakhshayesh@yahoo.com
}

Received 2016 December 21; Accepted 2017 February 08.

\begin{abstract}
Background: Observations on Tuberculosis HIV co-infection in addition to epidemiologic molecular studies have recently provided strong evidence for the state of immune system as the major determinant of the TB imaging spectrum. However, the presence of any correlation between radiographic findings and the degree of suppression in HIV positive patients still remains controversial. The present study aimed to investigate the TB radiographic manifestation in HIV positive patients and its relationship to the CD4 positive cell count.

Methods: Chest radiography of 15 HIV positive patients with a definite diagnosis of pulmonary Tuberculosis in Masih Daneshvari hospital, between 2013 - 2014, were retrospectively reviewed. Radiographic findings and severity were categorized as typical (upper lobe infiltration/cavity) and atypical (middle/lower lobe opacity, adenopathy, pleural effusion and normal X-ray). Demographics and CD4 positive cell count were also recorded. Data analysis was performed using SPSS version 23 (frequency and mean for descriptive quantitative variables and Logistic regression analysis for correlation, $\mathrm{P}<0.05$ ).

Results: Of a total 15 patients (86.7\% men and 13.3\% women), 78.6\% had CD4 positive counts below 350 ( $m e a n \pm S D ; 229.15 \pm 199.45)$. The most common radiographic findings in descending order of frequency were adenopathy (53.3\%), pleural effusion (26.7\%) and cavitation (6.7\%) with an overall atypical presentation of $93.3 \%$. This study failed to reveal any statistically significant correlation between CD4 positive cell count and radiographic manifestation as well as severity.

Conclusions: In CD4 positive cell count below 500, the dominant radiographic pattern of Tuberculosis is atypical presentation. At this level of immunity, CD 4 positive T cell dysfunction may play a deterministic role in TB radiographic manifestation.
\end{abstract}

This is an abstract presented in the 33rd Iranian congress of radiology (ICR) and the 15th congress of Iranian radiographic science association (IRSA). 